hep-ph/9506354

BA-95-15

UMD-PP-95-117

April 1995

\title{
New Vector-Scalar Contributions to Neutrinoless Double Beta decay and Constraints on R-Parity Violation
}

\author{
K.S. Babu円 \\ Bartol Research Institute \\ University of Delaware \\ Newark, DE 19716 \\ and \\ R.N. Mohapatra| \\ Department of Physics and Astronomy \\ University of Maryland \\ College Park, MD 20742
}

\begin{abstract}
We show that in the minimal supersymmetric standard model (MSSM) with $\mathrm{R}$-parity breaking as well as in the left-right symmetric model, there are new observable contributions to neutrinoless double beta decay $\left(\beta \beta_{0 \nu}\right)$ arising from hitherto overlooked diagrams involving the exchange of one $\mathrm{W}$ boson and one scalar boson. In particular, in the case of MSSM, the present experimental bounds on $\beta \beta_{0 \nu}$ lifetime improves the limits on certain $\mathrm{R}$-parity violating couplings by about two orders of magnitude. It is shown that similar diagrams also lead to enhanced rates for $\mu^{-} \rightarrow e^{+}$conversion in nuclei, which are in the range accessible to ongoing experiments.
\end{abstract}

\footnotetext{
${ }^{1}$ Address starting September 1995: School of Natural Sciences, Institute for Advanced Study, Olden Lane, Princeton, NJ 08540; work supported by the Department of Energy Grant \#DEFG02-91ER406267
}

${ }^{2}$ Work supported by the National Science Foundation Grant \#PHY-9119745 
With the standard model of electroweak interactions brilliantly confirmed by a host of experiments, the search is on for the next level of physics at $\mathrm{TeV}$ or higher scales. There exist many interesting scenarios which address the various naturalness problems of the standard model framework. A generic feature of many of these scenarios is that conservation laws present in the standard model no longer remain valid. A typical conservation law that breaks down is the one corresponding to lepton-number symmetry. To make any theoretical headway, one needs to know the strength of the lepton number non-conserving interactions as well as of any other kind of interactions that may accompany them.

It has been known for a long time [1] that neutrinoless double beta decay $\left(\beta \beta_{0 \nu}\right)$ is a very sensitive probe of lepton number violating terms in the Lagrangian such as the Majorana mass of the light neutrinos [2], right-handed weak couplings involving heavy Majorana neutrinos [3,4], as well as Higgs [5] and other interactions such as those involving $\mathrm{R}$-parity breaking in the supersymmetric model [6]. The reason why this observation is interesting is that, the steadily improving experimental limits [7] on $\beta \beta_{0 \nu}$ life-time can then be translated into more stringent limits [8] on the parameters of these new physics scenarios. This is an extremely valuable information to have in our search for physics beyond the standard model.

It is the goal of this letter to point out another class of hitherto unnoticed contributions to $\beta \beta_{0 \nu}$ decay in the following two classes of theories: (i) Minimal supersymmetric models (MSSM) with R-parity violation; (ii) Left-right symmetric models with a low mass $W_{R}$. These new contributions are of vector-scalar type in that they involve the exchange of a $W_{L}$ together with a charged scalar boson with a virtual light neutrino. These contributions do not involve a helicity flip of the internal light neutrino, and therefore, their amplitudes are enhanced relative to the ordinary contribution proportional to the light Majorana neutrino mass by roughly a factor of $p_{F} / m_{\nu} \sim(100 \mathrm{MeV} / 1 \mathrm{eV}) \sim 10^{8}\left(p_{F}\right.$ is the Fermi momentum of the nucleons in the nuclei). Turning the argument around, the strength of such lepton number violating scalar boson interactions can be constrained to be $G_{e f f} \leq 10^{-8} G_{F}$ from the $(\beta \beta)_{0 \nu}$ process (modulo nuclear matrix element uncertainties), which indeed is a severe constraint. The effect turns out to be most dramatic on certain Rviolating couplings in the MSSM in that it directly involves the R-violating couplings and superpartner masses. In the left-right models, although such contributions involve unknown Higgs boson mixings, they do lead to interesting bounds for certain range of values for these parameters. We also point out that similar enhanced vectorscalar contributions exist in other rare processes such as $\mu^{-} \rightarrow e^{+}$conversion in nuclei which turn out to be in the experimentally accessible range.

The new contributions arise from the combination of two effective four-Fermi interactions of the following type which as we will show later can arise in several 
interesting classes of gauge models:

$$
\begin{aligned}
H_{e f f}= & \frac{G_{F}}{\sqrt{2}}\left(\bar{e} \gamma_{\mu}\left(1-\gamma_{5}\right) \nu_{e} \bar{u} \gamma^{\mu}\left(1-\gamma_{5}\right) d+\epsilon_{1}^{e e} \bar{d}\left(1-\gamma_{5}\right) u \nu_{e}^{T} C^{-1}\left(1-\gamma_{5}\right) e+\right. \\
& \left.\epsilon_{2}^{e e} \bar{d}\left(1-\gamma_{5}\right) \nu_{e} u^{T} C^{-1}\left(1-\gamma_{5}\right) e\right)+ \text { h.c. }
\end{aligned}
$$

In the above, the first term is the usual ( $\mathrm{V}-\mathrm{A})$ interaction, the other two are effective lepton number violating terms. In order to evaluate the matrix elements between nuclear states, we need to do Fierz reordering of the $\epsilon_{2}^{e e}$ term, which casts it in the form:

$$
\frac{G_{F}}{2 \sqrt{2}} \epsilon_{2}^{e e}\left(\bar{d}\left(1-\gamma_{5}\right) u \overline{e^{c}}\left(1-\gamma_{5}\right) \nu_{e}+\frac{1}{2} \bar{d} \sigma^{\mu \alpha}\left(1-\gamma_{5}\right) u \overline{e^{c}}\left(1-\gamma_{5}\right) \sigma_{\mu \alpha} \nu_{e}\right) !
$$

The parameters $\epsilon_{i}^{e e}(i=1,2)$ characterize the new interactions that arise in a gauge model so that any limit on them translates into limits on the parameters on the theory leading to this interaction.

It is easy to see that the two new interaction terms in Eq. (1) give contributions to $\beta \beta_{0 \nu}$ decay which do not depend on the neutrino mass and involve a vector current at one hadronic vertex and a scalar current in the other (hence the name vectorscalar; of course as just mentioned, the $\epsilon_{2}^{e e}$-type scalar interaction of Eq. (1) after Fierz reordering generates also a tensor coupling). The resulting effective $\Delta L=2$ Hamiltonian is then given in momentum space by:

$$
\begin{aligned}
H_{\Delta L=2} & =G_{F}^{2}\left(\left(\epsilon_{1}^{e e}+\frac{\epsilon_{2}^{e e}}{2}\right) \bar{u}\left(1+\gamma_{5}\right) d \bar{u} \gamma_{\mu}\left(1-\gamma_{5}\right) d \bar{e} \gamma^{\mu}\left(1-\gamma_{5}\right) \frac{1}{\gamma \cdot q} C \bar{e}^{T}\right. \\
& \left.+\frac{\epsilon_{2}^{e e}}{4} \bar{u} \sigma^{\lambda \alpha}\left(1+\gamma_{5}\right) d \bar{u} \gamma_{\mu}\left(1-\gamma_{5}\right) d \bar{e} \gamma^{\mu} \sigma_{\lambda \alpha}\left(1-\gamma_{5}\right) \frac{1}{\gamma \cdot q} C \bar{e}^{T}\right)+ \text { h.c. }
\end{aligned}
$$

In Eq. (2), q refers to the momentum of the internal light neutrino propagator. This effective Hamiltonian of course has to be evaluated between nuclear states. This gives rise to an effective neutrino potential as in the case of the neutrino mass contribution to $\beta \beta_{0 \nu}$ decay with the difference that $\left\langle m_{\nu} / q^{2}\right\rangle_{N u c l}$. gets replaced by $\left\langle\gamma \cdot q / q^{2}\right\rangle_{N u c l}$. (and a different operator for the tensor coupling). To the best of our knowledge, such nuclear matrix elements for $\beta \beta_{0 \nu}$ decay have not been evaluated in the literature. We therefore resort to a crude estimate and assume an average value of $q$ to be equal to the Fermi momentum $p_{F}$ of the nucleons in the nucleus $(\approx 100$ 
$\mathrm{MeV}$ ). The present upper limits on $m_{\nu}$ of about $1 \mathrm{eV}$ then translates to an upper limit on the new interaction parameter $\epsilon$ as follows:

$$
\epsilon_{1,2}^{e e} \leq 1 \times 10^{-8}
$$

We realize that due to the crudeness of our estimate of the nuclear matrix element, the above upper limit is likely to be uncertain by perhaps a factor of five or so. Nevertheless it is important to note that the limit on $\epsilon_{1,2}$, barring unforeseen nuclear suppressions, is rather stringent and will imply important restrictions on the parameters of the gauge models leading to Eq. (1). Let us therefore, proceed to the kind of gauge models where the last two terms in Eq. (1) can arise at low energies.

\section{MSSM with $R$-parity violation:}

As is well-known, the minimal supersymmetric standard model can have explicit [9] violation of the R-symmetry (defined by $(-1)^{3 B+L+2 S}$ ), leading to lepton number violating interactions in the low energy Lagrangian. The three possible types of couplings in the superpotential are [10]:

$$
W^{\prime}=\lambda_{i j k} L_{i} L_{j} E_{k}^{c}+\lambda_{i j k}^{\prime} L_{i} Q_{j} D_{k}^{c}+\lambda_{i j k}^{\prime \prime} U_{i}^{c} D_{j}^{c} D_{k}^{c}
$$

Here $L, Q$ stand for the lepton and quark doublet superfields, $E^{c}$ for the lepton singlet superfield and $U^{c}, D^{c}$ for the quark singlet superfields. $i, j, k$ are the generation indices and we have $\lambda_{i j k}=-\lambda_{j i k}, \lambda_{i j k}^{\prime}=-\lambda_{i k j}^{\prime}$. The $S U(2)$ and color indices in Eq. (4) are contracted as follows: $L_{i} Q_{j} D_{k}^{c}=\left(\nu_{i} d_{j}^{\alpha}-e_{i} u_{j}^{\alpha}\right) D_{k \alpha}^{c}$, etc. The simultaneous presence of all three terms in Eq. (4) will imply rapid proton decay, which can be avoided by setting the $\lambda^{\prime \prime}=0$. In this case, baryon number remains an unbroken symmetry while lepton number is violated [11].

There are two types of vector-scalar contributions to $\beta \beta_{0 \nu}$ and related $\Delta L=2$ processes. These are shown in Figures 1 and 2. The dominant contribution to $\beta \beta_{0 \nu}$ in this model arises from Fig. 1, where the exchanged scalar particles are the $\tilde{b}-\tilde{b}^{c}$ pair. This leads to a contribution to $\epsilon_{2}^{e e}$ given by

$$
\epsilon_{2}^{e e} \simeq\left(\frac{\left(\lambda_{113}^{\prime} \lambda_{131}^{\prime}\right)}{2 \sqrt{2} G_{F} M_{\tilde{b}}^{2}}\right)\left(\frac{m_{b}}{M_{\tilde{b}^{c}}^{2}}\right)\left(\mu \tan \beta+A_{b} m_{0}\right)
$$

Here $A, m_{0}$ are supersymmetry breaking parameters, while $\mu$ is the supersymmetric mass of the Higgs bosons. $\tan \beta$ is the ratio of the two Higgs vacuum expectation values and lies in the range $1 \leq \tan \beta \leq m_{t} / m_{b} \approx 60$. For the choice of all squark 
masses as well as $\mu$ and the SUSY breaking mass parameters being of order of $100 \mathrm{GeV}, A=1, \tan \beta=1$, we find from Eq. (5) that $\lambda_{113}^{\prime} \lambda_{131}^{\prime} \leq 3 \times 10^{-8}$, which is more stringent limit on this parameter than the existing ones [12]. The present limits on these parameters are $\lambda_{113}^{\prime} \leq 0.03, \lambda_{131}^{\prime} \leq 0.26$, which shows that the bound derived here from $\beta \beta_{0 \nu}$ is about five orders of magnitude more stringent on the product $\lambda_{113}^{\prime} \lambda_{131}^{\prime}$. If the exchanged scalar particles in Fig. 1 are the $\tilde{s}-\tilde{s}^{c}$ pair, $b$ gets replaced by $s$ in Eq.(5) and one obtains a limit $\lambda_{121}^{\prime} \lambda_{112} \leq 1 \times 10^{-6}$, which also is more stringent by about four orders of magnitude than existing limits $\left(\lambda_{121}^{\prime} \leq 0.26, \lambda_{112}^{\prime} \leq 0.03\right)$. We note that the gluino exchange diagram $[6,8]$ discussed in the context of $\beta \beta_{0 \nu}$ only constrains the parameter $\lambda_{111}^{\prime}$, while the vector-scalar exchange graphs constrain several other couplings.

The diagram in Fig. 2, due to the antisymmetry of $\lambda_{i j k}$, does not contribute to $\beta \beta_{0 \nu}$ decay, but will be important for $\mu^{-} \rightarrow e^{+}$conversion (see discussions below).

Let us also note that there exist indirect limits on the $\lambda$ and $\lambda^{\prime}$ couplings arising from the induced neutrino masses. The magnitudes of these masses are given generically by $m_{i j}^{\nu} \sim \lambda_{i k l}^{\prime} \lambda_{j k l}^{\prime} m_{k} m_{l} /\left(16 \pi^{2} M_{\tilde{q}}\right)$, where $m_{k, l}$ stand for the masses of the $k$ th and $l$ th down quarks (with a similar expression for the $\lambda$ couplings). The couplings $\lambda_{i 33}^{\prime} \lambda_{j 33} \leq 6 \times 10^{-5}$ is the most severely constrained (being proportional to the $b$-quark mass-squared) where the induced neutrino mass has been assumed to be $\leq 100 \mathrm{eV}$, which is cosmologically safe. The corresponding limit on $\lambda_{131}^{\prime} \lambda_{113}^{\prime} \leq .24$ (using $m_{\nu} \leq 1 \mathrm{eV}$ ) is a trivial constraint to be compared with the $\beta \beta_{0 \nu}$ decay limit derived here.

Constraints on the Left-right symmetric model:

Let us consider the minimal left-right symmetric model with a see-saw mechanism for neutrino masses [13]. The gauge group of the model is $S U(3)_{C} \times S U(2)_{L} \times$ $S U(2)_{R} \times U(1)_{B-L}$. The Higgs sector of the model consists of the bi-doublet field $\phi \equiv(1 / 2,1 / 2,0)$ and triplet Higgs fields: $\Delta_{L}(1,0,+2) \oplus \Delta_{R}(0,1,+2)$. The Yukawa couplings which are invariant under gauge and parity symmetry can be written as:

$$
\begin{aligned}
\mathcal{L}_{Y}= & \bar{\Psi}_{L} h^{\ell} \phi \Psi_{R}+\bar{\Psi}_{L} \tilde{h}^{\ell} \tilde{\phi} \Psi_{R}+\bar{Q}_{L} \phi h^{q} Q_{R}+\bar{Q}_{L} \tilde{h}^{q} \tilde{\phi} Q_{R}+ \\
& \Psi_{L}^{T} \tau_{2} \vec{\tau} \cdot f \vec{\Delta}_{L} C^{-1} \Psi_{L}+L \rightarrow R+h . c .
\end{aligned}
$$

where $h, \tilde{h}$ are hermitian matrices while $f$ is a symmetric matrix in the generation space. $\Psi$ and $Q$ here denote the leptonic and quark doublets respectively.

The gauge symmetry is spontaneously broken by the vacuum expectation values: $\left\langle\Delta_{R}^{0}\right\rangle=V_{R} ;\left\langle\Delta_{L}^{0}\right\rangle \simeq 0 ;$ and $\langle\phi\rangle=\operatorname{diag} .\left(\kappa, \kappa^{\prime}\right)$. As usual, $\langle\phi\rangle$ gives masses to the charged fermions and Dirac masses to the neutrinos whereas $\left\langle\Delta_{R}^{0}\right\rangle$ leads to the see-saw mechanism for the neutrinos in the standard way [13]. 
The physics we are interested in comes from the left-handed triplet sector of the theory through its mixing with the bidoublet field which arise from the couplings in the Higgs potential, such as $\operatorname{Tr}\left(\Delta_{L} \phi \Delta_{R}^{\dagger} \phi^{\dagger}\right)$ after the full gauge symmetry is broken down to $U(1)_{\mathrm{em}}$. Specifically, there is a mixing between the singly charged components of $\phi$ and $\Delta_{L}$ [14] (we denote this mixing term by an angle $\theta$ ). This will contribute to the four-Fermi interaction of the form given by the $\epsilon_{1}^{e e}$ term through the diagram shown in Fig. 3 with

$$
\epsilon_{1}^{e e} \simeq \frac{h_{u} f_{11} \sin 2 \theta}{4 \sqrt{2} G_{F} M_{H^{+}}^{2}}
$$

where we have assumed that $H^{+}$is the lighter of the two Higgs fields. We get $h_{u} f_{11} \sin 2 \theta \leq 6 \times 10^{-9}\left(M_{H^{+}} / 100 \mathrm{GeV}\right)^{2}$, which is quite a stringent constraint on the parameters of the theory. To appreciate this somewhat more, we point out that one expects $h_{u} \approx m_{u} / m_{W} \approx 5 \times 10^{-5}$ in which case, we get an upper limit for the coupling of the Higgs triplets to leptons $f_{11} \sin 2 \theta \leq 10^{-4}$ (for $m_{H^{+}}=100 \mathrm{GeV}$ ). Taking a reasonable choice of $\theta \sim M_{W_{L}} / M_{W_{R}} \sim 10^{-1}$ would correspond to a limit $f_{11} \leq 10^{-3}$. Limits on this parameters from analysis [15] of Bhabha scattering is only of order .2 or so for the same value of the Higgs mass.

$\mu^{-} \rightarrow e^{+}$conversion:

Another class of rare processes where the new vector-scalar contribution makes an important impact is the process of $\mu^{-} \rightarrow e^{+}$conversion in nuclei which arises with an observable strength in the $\mathrm{R}$ violating MSSM. This involves the couplings $\lambda_{i j k}$ which were not constrained by the considerations of neutrinoless double beta decay due to anti-symmetry of the Yukawa couplings. Another contribution involves a different product of $\lambda^{\prime}$ couplings than what appeared in the $\beta \beta_{0 \nu}$ process. We parametrize the effective four Fermi interaction for this process in analogy to Eq. (1) with $\epsilon_{1}^{e e}$ replaced by two terms $\epsilon_{1}^{e \mu}$ and $\epsilon_{1}^{\mu e}$ and similarly for $\epsilon_{2}$. Here $\epsilon_{1}^{e \mu}$ is the coefficient of the term $G_{F} / \sqrt{2}\left[\bar{d}\left(1-\gamma_{5}\right) u \nu_{e}^{T} C^{-1}\left(1-\gamma_{5}\right) \mu\right]$ etc. The effective strengths of these couplings arising from Fig. 2 (as well as from Fig. 1 with $e$ replaced by a $\mu$ ) are found to be

$$
\begin{aligned}
\epsilon_{1}^{e \mu} & =\frac{\lambda_{123} \lambda^{\prime} \_311}{2 \sqrt{2} G_{F} m_{\tilde{\tau}}^{2} m_{\tilde{\tau}^{c}}^{2}} m_{\tau}\left(\mu \tan \beta+A_{\tau} m_{0}\right) \\
\epsilon_{2}^{e \mu} & =\frac{\lambda_{213}^{\prime} \lambda_{131}^{\prime}}{2 \sqrt{2} G_{F} m_{\tilde{b}}^{2} m_{\tilde{b}^{c}}^{2}} m_{b}\left(\mu \tan \beta+A_{b} m_{0}\right)
\end{aligned}
$$


with similar expressions for $\epsilon_{1,2}^{\mu e}$. The existing limits on the $\lambda^{\prime}$ in Eq. (8) are $\lambda_{213}^{\prime} \leq$ $0.09, \lambda_{131}^{\prime} \leq 0.26$, so that for the squark masses of order $100 \mathrm{GeV}, \epsilon_{2}^{e \mu}$ can be as large as $10^{-1}$ or so. The branching ratio for $\mu^{-} \rightarrow e^{+}$conversion relative to $\mu$-capture can be obtained by a naive scaling by the nucleon mass and we find $\operatorname{Br}\left(\mu^{-} \rightarrow e^{+}\right) \simeq 10^{-12}$ for this choice of $\epsilon_{2}^{e \mu}$. It is interesting that this is in the accessible range of the current experiments. The sensitivity of these experiments is expected to improve by another order of magnitude in the near future [16].

We note that the corresponding predictions for the left-right model is down by several orders of magnitude.

In summary, we have discussed a new class of contributions to the double lepton number violating processes which may arise in several extensions of the standard model. In particular, we find that the existing experimental limits on neutrinoless double beta decay lead to very stringent constraints on the $\mathrm{R}$-violating couplings in MSSM as well as the lepton number violating Higgs couplings of the left-right symmetric model. Furthermore, we find the exciting possibility that for presently allowed range of parameters in the MSSM, $\mu^{-} \rightarrow e^{+}$conversion in nuclei is in the observable range. While we have focussed on only two classes of models, our results are more general and should apply to other schemes, such as $L$-violating leptoquark models. 


\section{References}

1. W. C. Haxton and G. Stephenson, Prog. in Part. and Nucl. Phys. 12, 409 (1984); H. Grotz and H. Klapdor, The Weak Interactions in Nuclear, Particle and Astrophysics, Adam Hilger,Bristol, (1990); R. N. Mohapatra and P. B. Pal, Massive Neutrinos in Physics and Astrophysics, World scientific, Singapore (1991); M. Moe and P. Vogel, Ann. Rev. Nucl. and Part. Sc., 44, 247 (1994).

2. M. Doi, T. Kotani, H. Nishiura and E. Takasugi, Prog. Theor. Phys. Suppl. 83, 1 (1985).

3. A. Halprin, P. Minkowski, H. Primakoff and S.P. Rosen, Phys. Rev. D13, 2567 (1976).

4. R.N. Mohapatra, Phys. Rev. D34, 909 (1986).

5. R.N. Mohapatra and J. Vergados, Phys. Rev. Lett. 47, 1713 (1981); J. Schecter and J.W.F. Valle, Phys. Rev. D25, 2951 (1982); W.C. Haxton, S.P. Rosen and G.J. Stephenson, ibid., D26,1805 (1982); L. Wolfenstein, ibid., D26, 2507 (1982).

6. R. N. Mohapatra, Phys. Rev. D34, 3457 (1986).

7. H. V. Klapdor-Kleingrothaus, Prog. in Part. and Nucl. Phys. 32, 261 (1994); A. Balysh et. al., Phys. Lett. B (to appear).

8. J. D. Vergados, Phys. Lett. B184, 55 (1987); M. Hirsch, H. V. KlapdorKleingrothaus and S. G. Kovalenko, Heidelberg Preprint (1995).

9. L. Hall and M. Suzuki, Nucl. Phys. B231, 419 (1984).

10. In addition, one can write a term $\mu_{i} L_{i} H_{u}$ in $W^{\prime}$. Although this term can be rotated away by a field redefinition, the VEVs of the scalar neutrinos cannot be set to zero in general then. In supergravity models, however, such VEVs get induced only by the effect of large Yukawa couplings, in which case the sneutrino VEVS will remain zero.

11. There is also the possibility of setting $\lambda=\lambda^{\prime}=0$, with $\lambda^{\prime \prime} \neq 0$, in which case $B$ is violated, but $L$ is not. We shall not pursue this alternative here.

12. V. Barger, G. Giudice and T. Han, Phys. Rev. D40, 2987 (1989).

13. R.N. Mohapatra and G. Senjanović, Phys. Rev. Lett. 44, 912 (1980); Phys. Rev. D23, 165 (1981). 
14. Note that in the limit $\kappa^{\prime}=0$, this coupling does not induce a $\left\langle\Delta_{L}\right\rangle$. See K.S. Babu and R.N. Mohapatra, Phys. Rev. Lett. 64, 9 (1990).

15. M. Schwarz, Phys. Rev. D40, 1521 (1989).

16. W. Bertl, private communication.

\section{Figure Caption:}

Fig.1: The dominant diagram contributing to $\epsilon_{2}^{e e}$ type Four-Fermi term (Eq. (1)) in the supersymmetric model.

Fig.2: The diagram contributing to $\epsilon_{1}^{e \mu}$ type Four-Fermi terms relevant for $\mu^{-} \rightarrow e^{+}$ conversion in the supersymmetric model.

Fig.3: The vector-scalar exchange diagram for $\beta \beta_{0 \nu}$ in the left-right symmetric model. 
This figure "fig1-1.png" is available in "png" format from: http://arxiv.org/ps/hep-ph/9506354v1 
This figure "fig1-2.png" is available in "png" format from: http://arxiv.org/ps/hep-ph/9506354v1 